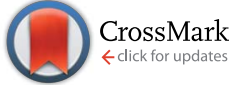

Cite this: RSC Adv., 2017, 7, 17154

\title{
Hierarchically templated beads with tailored pore structure for phosphopeptide capture and phosphoproteomics $\dagger$
}

\author{
Celina Wierzbicka, ${ }^{a}$ Silje B. Torsetnes, ${ }^{b}$ Ole N. Jensen, ${ }^{b}$ Sudhirkumar Shinde ${ }^{a}$ \\ and Börje Sellergren ${ }^{\star a}$
}

\begin{abstract}
Two templating approaches to produce imprinted phosphotyrosine capture beads with a controllable pore structure are reported and compared with respect to their ability to enrich phosphopeptides from a tryptic peptide mixture. The beads were prepared by the polymerization of urea-based host monomers and crosslinkers inside the pores of macroporous silica beads with both free and immobilized template. In the final step the silica was removed by fluoride etching resulting in mesoporous polymer replicas with narrow pore size distributions, pore diameters $\approx 10 \mathrm{~nm}$ and surface area $>260 \mathrm{~m}^{2} \mathrm{~g}^{-1}$. The beads displayed pronounced phosphotyrosine affinity and selectivity in binding tests using model peptides in acetonitrile rich solutions with a performance surpassing solution polymerized bulk imprinted materials. Tests of the beads for the enrichment of phosphopeptides from tryptic digests of twelve proteins revealed both $\mathrm{pY} / \mathrm{pS}$ and $\mathrm{pY} / \mathrm{Y}$ selectivity. This was reflected in a nearly 6 -fold increase in the enrichment factor of a 23-mer $\mathrm{pY}$-peptide and $\mathrm{pY} / \mathrm{pS}$ normalized intensity ratios up to 1.5, when comparing the template mesoporous beads with the bulk materials.
\end{abstract}

Received 10th January 2017

DOI: $10.1039 / c 7 r a 00385 d$

rsc.li/rsc-advances

\section{Introduction}

Protein phosphorylation is a reversible posttranslational modification (PTM) playing a central role in numerous biological events, several of which are directly linked to disease pathogenesis. ${ }^{1}$ Phosphoproteomics, i.e. the dynamic mapping of the occurrence of this PTM, has become an essential activity in the development of new drugs and diagnostic methods. ${ }^{2-7}$ Difficulties in analysis of phosphorylated proteins arise from their low abundance (e.g. phosphotyrosine) and transient nature. These challenges were addressed in functional proteomics using phosphopeptide specific affinity enrichment techniques and mass spectrometry, recently in parallel high throughput formats. ${ }^{4,7}$ Several chemoaffinity-based techniques to concentrate phosphopeptides from complex biological samples have been developed to date, each with their benefits and limitations, the latter generally referring to a lack of side chain selectivity i.e. recognition of $\mathrm{pY}, \mathrm{pS}$ and $\mathrm{pT}$ respectively, a bias towards certain amino acids or sequence motifs and

${ }^{a}$ Department of Biomedical Sciences, Faculty of Health and Society, Malmö University, SE 20506 Malmö, Sweden. E-mail: borje.sellergren@mah.se

${ }^{b}$ Department of Biochemistry and Molecular Biology and VILLUM Center for Bioanalytical Sciences, University of Southern Denmark, DK-5230 Odense M, Denmark $\dagger$ Electronic supplementary information (ESI) available: Binding isotherms, SEM images and optical micrographs, nitrogen sorption, TGA and elemental analysis, ATR-FTIR and MALDI spectra, MALDI peak intensity analysis. See DOI: 10.1039/c7ra00385d incompatibility with high throughput formats. ${ }^{3}$ Another limitation is inherent in the bottom up proteomics approach per se. ${ }^{8}$ Since this relies on the digestion of proteins to small peptides ( $<3 \mathrm{kDa})$ by trypsin followed by enrichment and LC-MS based readout of the peptide sequences, it is difficult to pinpoint multiple modifications (e.g. histone acetylation, methylation, phosphorylation) that are far apart from each other. Top down or middle down proteomics analyzing intact proteins or partially digested proteins (3-20 kDa) respectively address these issues. ${ }^{8}$ Enrichment techniques adapted to larger phosphopeptide targets are therefore needed.

Molecular epitope imprinting has in the above contexts emerged as a possible alternative affinity technique. ${ }^{9}$ Several approaches to develop phosphopeptide selective molecularly imprinted polymers (MIPs) have been reported to date. ${ }^{9-14}$ In our first report, we introduced an epitope imprinting approach relying on the templating of minimal fragments of the targeted peptides. ${ }^{9}$ Hence, imprinting of N,C-protected phosphoaminoacids such as pY and pS produced binding sites complementary to $\mathrm{pY}$ and $\mathrm{pS}$ peptides respectively. The success of this approach depended on the combined use of lipophilic phosphoamino acid salts and urea based host monomer in a $1: 2$ stoichiometry. Incorporation of the resulting ternary complexes in the polymer scaffold followed by template removal left behind binding sites capable of binding the phosphoamino acid guest by quadrupole hydrogen bonding.

Having addressed the molecular recognition properties of these materials we have recently turned our attention to their 
morphological properties. The first generation materials were prepared as porous monoliths following the classical approach to produce macroporous polymers. ${ }^{15}$ This results in materials displaying a wide distribution of pore sizes with a significant number of pores in the micro- and low meso-porous range. As a result, the inner pores of such materials show a size dependent binding preference and are likely to be poorly accessible to larger sized peptides. This will compromise their use for unbiased phosphopeptide enrichment and especially for middle down proteomics. In order to address this weakness, approaches to control the pore sizes and their distribution are required.

Grafting of thin polymer films or templated synthesis using inorganic supports of known morphology are very promising in this regard. ${ }^{\mathbf{1 6 - 1 8}}$ A prominent example is porous silica which is commonly used as a sacrificial template for the synthesis of mesoporous organic polymer networks with defined particle size, shape and porosity. Hierarchical imprinting takes advantage of this morphology control to obtain imprinted polymer beads exhibiting highly accessible binding sites. ${ }^{19}$ The molecular template can either be immobilized on the walls ${ }^{\mathbf{1 9}}$ of the mold or the template can be simply dissolved in the monomer mixture. ${ }^{20}$ This gives rise to polymer beads featuring enhanced binding site accessibility and faster mass transfer in chromatography. We have here assessed the latter two approaches as means to reduce the size bias in pY selective phosphopeptide enrichments. Micron sized mesoporous polymer beads were thus prepared and compared with a bulk-imprinted benchmark in a mass spectrometry assay of the enrichment of phosphopeptides from tryptic peptide mixtures. We hypothesized that hierarchical imprinting can generate efficient affinity capture for phosphopeptides, particularly of larger phosphopeptides surpassing $3 \mathrm{kDa}$ that are of interest in studies of PTM crosstalk. Our results demonstrate the feasibility of this approach to boost both overall phospho-peptide and pY selectivity.

\section{Experimental}

\section{Materials}

Pentaerythritol triacrylate (PETA) was from Polysciences (Warrington, PA, USA). $\quad N, N^{\prime}$-azo-bis(2,4-dimethyl)valeronitrile (ABDV) was from Wako Chemicals $\mathrm{GmbH}$ (Neuss, Germany); 1-hydroxybenzotriazole (HOBt), 1,2,2,6,6-pentamethylpiperidine (PMP), triethylammonium bicarbonate (TEAB) buffer (1 $\mathrm{M})$, acetic anhydride $\left(\mathrm{Ac}_{2} \mathrm{O}\right)$ and formic acid (FA) were from Fluka (Deisenhofen, Germany). Trifluoroacetic acid (TFA), acetonitrile (ACN), methanol $(\mathrm{MeOH})$ were from VWR chemicals. Dry dichloromethane (dry DCM), triethylamine (TEA), ammonium hydrogen difluoride $\left(\mathrm{NH}_{4} \mathrm{HF}_{2}\right)$, 2,5-dihydroxybenzoic acid (DHB), piperidine, ninhydrin, acrylamide, iodoacetamide (IAA) and 1,4-dithiothreitol (DTT) were from Sigma-Aldrich (Milwaukee, USA). Dimethylformamide (DMF), dry acetonitrile (dry ACN) were from Acros Organics. Fmoc-pTyr$\mathrm{OH}$, Fmoc-pSer-OH, Fmoc-Tyr-OH and Fmoc-Gly-OH were from Bachem GmbH (Weil am Rhein, Germany). Fmoc$\operatorname{Tyr}\left(\mathrm{PO}\left(\mathrm{NMe}_{2}\right)_{2}\right)-\mathrm{OH}, \quad N$-ethyldiisopropylamine (DIPEA), (benzotriazol-1-yloxy)tripyrrolidinophosphonium hexafluoro- phosphate (PyBOP) and dichloromethane (DCM) were from Merck KGaA (Dramstadt, Germany). Empore C8 extraction disk was from 3M Bioanalytical Technologies (St. Paul, MN, USA). Trypsin was from Promega (Madison, WI, USA).

Proteins and peptides. The twelve proteins used for protein digestion were: carbonic anhydrase (bovine), ribonuclease $\mathrm{B}$ (bovine pancreas), serum albumin (bovine), lactoglobulin (bovine), $\alpha$-casein (bovine), $\beta$-casein (bovine), ovalbumin (chicken), lysozyme (chicken), alcohol dehydrogenase (Baker's yeast), myoglobin (whale skeletal muscle), $\alpha$-amylase (Bacillus species) and transferrin (human). Transferrin was from ACE Biosciences A/S (Hilleroed, Denmark), other proteins were from Sigma-Aldrich (Milwaukee, USA). Phosphopeptides YSSDPTGALTEDSIDDTFLPVPEPYINQSVPK, RPAGSVQNPVPYHNQPLNPAPSRD, GSHQISLDNPDPYQQDFFPK, MHLPSPTDSNFPYR and GSTAENAEPYLR were custom synthesized by GenicBio (Shanghai, China). GADDSpYpYTAR, GADDSYpYTAR, GADDSYYTAR, DRVPYIHPF, DRVPSIHPF, VPYI, VpSI and VYI were custom synthesized by LifeTein LLC (Hillsborough, NJ, USA). DRVYIHPF was from Fluka (Deisenhofen, Germany).

Amino-functionalized macroporous silica beads ( $\left.\mathrm{NH}_{2} @ \mathrm{Si}\right)$ with an average particle size of $30 \mu \mathrm{m}$, a surface area $(S)$ of $45 \mathrm{~m}^{2}$ $\mathrm{g}^{-1}$, average pore diameter $\left(D_{\mathrm{p}}\right)$ of $47.5 \mathrm{~nm}$, and a pore volume $\left(V_{\mathrm{p}}\right)$ of $0.81 \mathrm{~mL} \mathrm{~g}^{-1}$ were purchased from Fuji Silysia Chemical Ltd. (Kozoji-cho, Kasugai Aichi, Japan). $N$-(9-Fluorenylmethoxycarbonyl)- $O^{\prime}$-phosphonotyrosine ethyl ester (Fmoc-pTyr-OEt, 2) ${ }^{9}$ and $N$-3,5-bis(trifluoromethyl)-phenyl- $N^{\prime}$-4-vinylphenylurea $(\mathbf{1})^{21}$ were synthesized as reported elsewhere.

\section{Apparatus and methods}

High performance liquid chromatography (HPLC). HPLC measurements were carried out on Alliance 2795 instrument equipped with 2996 PDA detector (Waters, Milford, MA, USA).

Scanning electron microscopy (SEM). The particle morphology, size and size distribution were determined using Zeiss EVO LS 10 (E)SEM (Carl Zeiss AG, Oberkochen, Germany) at $T=25{ }^{\circ} \mathrm{C}, \mathrm{EHT}=15 \mathrm{kV}, \mathrm{WD}=4.5 \mathrm{~mm}$.

Optical microscopy. Optical micrographs were acquired using Nikon Optiphot Epi-Fluorescence microscope equipped with polarizing filters, phase contrast and a DS-U1 digital camera.

Thermogravimetric analysis (TGA). TGA was carried out using a TGAQ500 (TA Instruments). The sample (5-10 mg) was placed in a platinum pan, which is suspended in a sensitive balance together with the reference pan. The sample was then heated in a furnace with a heating rate of $20^{\circ} \mathrm{C} \mathrm{min}{ }^{-1}$, under $\mathrm{N}_{2}$ atmosphere.

Elemental analysis. Carbon, nitrogen, and hydrogen contents were determined by elemental analysis at the Department of Organic Chemistry, Johannes Gutenberg Universität Mainz using a Heraeus CHN-rapid analyzer (Hanau, Germany).

FT-IR spectroscopy. Infrared spectra were recorded using a Thermo Nicolet Nexus 6700 instrument (Thermo Scientific, Waltham, MA, USA).

UV absorbance measurements. UV absorbance measurements were performed on a Safire plate reader (Tecan Group 
Ltd., Männedorf, Switzerland) using a quartz 96-well microplate (Hellma GmbH, Müllheim, Germany).

MALDI-TOF-MS. All mass spectra were obtained using a MALDI reflector time of flight mass spectrometer (ultrafleXtreme MALDI TOF/TOF MS/MS; Bruker Daltonics, Bremen, Germany) controlled by flexControl software (version 2.4, Bruker Daltonics, Bremen, Germany). The system was operated in positive ion reflector mode only recording MS1 spectra in the $\mathrm{m} / \mathrm{z}$ range of 700-4000. 2,5-Dihydroxybenzoic acid (DHB, $40 \mathrm{mg}$ $\mathrm{mL}^{-1}$ ) in $50 \%$ ACN and $1 \%$ phosphoric acid was used as matrix. $^{22}$ Relative laser fluence was set at 55\%. Signals from 1000 laser shots $(10 \times 100$ shots at 10 different positions $)$ were averaged. Data collection, in terms of the scanning conditions and number of the scans, was performed identically for all samples unless otherwise noted. Mass spectrometric data analysis was performed using FlexAnalysis 3.4 software (Bruker Daltonik GmbH, Bremen, Germany). The signal intensity threshold for identification of peptides in the sample was arbitrary set at 9000 a.u. For the peptide YSSDPTGALTEDSIDDTFLPVPEPYINQSVPK threshold value was set at 3000 a.u.

Nitrogen sorption. Nitrogen sorption measurements were performed on the ASAP2020 Sorption Analyzer (Micrometrics, Norcross, GA, USA). Prior to the measurements, samples (100$150 \mathrm{mg})$ were heated at $105^{\circ} \mathrm{C}$ under high vacuum $\left(10^{-5} \mathrm{~Pa}\right)$ for $8 \mathrm{~h}$. The specific surface areas $\mathrm{S}$ were evaluated by using the Brunauer-Emmett-Teller (BET) method, the specific pore volumes $V_{\mathrm{p}}$ and the average pore diameter $D_{\mathrm{p}}$ by using the Barrett-Joiner-Halenda (BJH) theory applied to the desorption branch of the isotherm.

Measurement of swelling. NMR tubes were filled with dry polymer particles $(100 \mathrm{mg})$ and the height of dry polymer bed was measured. Next, ACN (1 mL) was added and the particles allowed to soak in the solvent for $24 \mathrm{~h}$. The particles were then allowed to settle and the bed height of the swollen particles was measured. The swelling factor was calculated as the ratio of the bed height of the swollen particles to the bed height of the dry particles.

\section{Silica surface modification}

$\mathrm{NH}_{2}$ @Si (20 g) was suspended in DMF (100 mL) in a $250 \mathrm{~mL}$ round bottomed flask equipped with an overhead stirrer. Next, acetic anhydride was added $(20 \mathrm{~mL})$ and the suspension was stirred at room temperature overnight. Thereafter the silica was filtered off, washed with DMF $(3 \times 50 \mathrm{~mL})$ and $\mathrm{MeOH}(3 \times 50$ $\mathrm{mL}$ ) and dried in vacuum overnight to yield $N$-acetylated silica (AcNH@Si). Negative ninhydrin test confirmed complete protection of the surface amino groups. The degree of acetylation was confirmed by TGA analysis.

\section{Immobilization of pY-template on silica}

The pY template was immobilized on the silica surface in a three step process (Fig. $\mathrm{S} 1 \dagger$ ).

Coupling. $\mathrm{NH}_{2} @ S i(3.0 \mathrm{~g})$ was suspended in DMF/DCM (50/ $50 \mathrm{v} / \mathrm{v} ; 30 \mathrm{~mL}$ ) under nitrogen in a two neck $100 \mathrm{~mL}$ round bottomed flask equipped with an overhead stirrer, next Fmoc$\operatorname{Tyr}\left(\mathrm{PO}\left(\mathrm{NMe}_{2}\right)_{2}\right)-\mathrm{OH}$ (1.0 eq.), PyBOP (1.1 eq.), HOBt (1.1 eq.) and
DIPEA (2.2 eq.) were added according to desired amount of template loading on silica surface (see Table $\mathrm{S} 1 \uparrow$ for the specific amounts used in each case). The reaction mixture was stirred under nitrogen atmosphere overnight. The silica was then filtered off and washed on a glass funnel with DMF $(3 \times 10 \mathrm{~mL})$ and DCM $(3 \times 10 \mathrm{~mL})$ and dried in vacuo.

Endcapping. The modified silica from the previous step $(2.5$ g) was suspended in DMF $(30 \mathrm{~mL})$ in a $100 \mathrm{~mL}$ round bottomed flask equipped with an overhead stirrer. After addition of acetic anhydride $(2 \mathrm{~mL})$ the reaction mixture was stirred at room temperature for $4 \mathrm{~h}$. Next, the silica was filtered off and washed with DMF $(3 \times 10 \mathrm{~mL})$ and $\mathrm{MeOH}(3 \times 10 \mathrm{~mL})$ and finally dried in vacuo to give Fmoc-p*Tyr@Si. Ninhydrin tests indicated that the protection of the amino groups was complete.

Deprotection. The silica from the previous step $(2.0 \mathrm{~g})$ was suspended in TFA/ $\mathrm{H}_{2} \mathrm{O}(9: 1 \mathrm{v} / \mathrm{v} ; 5 \mathrm{~mL})$ and shaken in a sealed $20 \mathrm{~mL}$ scintillation glass vial overnight. The solid was thereafter filtered off, washed with water $(3 \times 10 \mathrm{~mL})$ and $\mathrm{MeOH}(3 \times 10$ $\mathrm{mL}$ ) and dried in vacuo to yield silica with immobilized template Fmoc-pTyr@Si (Fmoc-pTyr@Si-A-Fmoc-pTyr@Si-E, Table S1†).

Quantification of immobilized template. $10 \mathrm{mg}$ of silica containing immobilized template (Fmoc-pTyr@Si-A-FmocpTyr@Si-E, Table S1 $\dagger$ ) was mixed with $1 \mathrm{~mL}$ of $20 \%$ piperidine in DMF and shaken for $1 \mathrm{~h}$. Next, the sample was centrifuged and the supernatant analyzed by UV absorbance measurements using a microplate reader $(\lambda=301 \mathrm{~nm})$. The amount of immobilized template was calculated using FmocGly-OH as an external standard.

\section{Preparation of pY-imprinted polymers}

Crushed monoliths (MIP-B, NIP-B). Fmoc-pTyr-OEt template (2) (100 $\mathrm{mg}, 0.20 \mathrm{mmol}$ ) was dissolved in dry ACN $(2.4 \mathrm{~mL})$ in a $20 \mathrm{~mL}$ scintillation glass vial. Next, PMP $(71 \mu \mathrm{L}, 0.40 \mathrm{mmol})$, functional monomer 1 (146 mg, $0.40 \mathrm{mmol}$ ), acrylamide $(28 \mathrm{mg}$, $0.40 \mathrm{mmol})$, PETA $(1.55 \mathrm{~g}, 5.20 \mathrm{mmol})$ and ABDV $(1 \% \mathrm{w} / \mathrm{w}$ of total monomers) were added to the solution. A sample of this prepolymerization mixture $(750 \mu \mathrm{L})$ was saved for subsequent preparation of the polymer beads. Remaining mixture was cooled on ice bath while bubbled with $\mathrm{N}_{2}$ for $10 \mathrm{~min}$ and transferred to a $50 \mathrm{~mL}$ Schlenk tube under continuous $\mathrm{N}_{2}$ flow. Next, the tube was closed with glass stopper and placed for $24 \mathrm{~h}$ in a water bath heated to $50^{\circ} \mathrm{C}$. The obtained polymer monolith (MIP-B) was removed from the tube, lightly crushed and washed with $\mathrm{MeOH} / 1 \mathrm{M} \mathrm{HCl}(80: 20 \mathrm{v} / \mathrm{v})(3 \times 30 \mathrm{~mL})$ followed by solvent extraction with $\mathrm{MeOH}$ in a Soxhlet apparatus for $24 \mathrm{~h}$. The course particles were then crushed and sieved. The fraction of particles with the size range $25-50 \mu \mathrm{m}$ was used in all experiments. Non-imprinted polymer (NIP-B) used as control was prepared in the same way but with the omission of template.

Polymer microspheres (MIP-M, NIP-M). Samples of AcNH@Si (1.0 g) were first deaerated in $50 \mathrm{~mL}$ Schlenk tubes (three cycles vacuum- $\mathrm{N}_{2}$ ) and then allowed to soak in MIP-B or NIP-B prepolymerization mixtures $(750 \mu \mathrm{L}$, vide supra) under continuous $\mathrm{N}_{2}$ flow while stirring with a spatula until the particles were freely flowing (indicating completion of the pore filling). Next, the tubes were closed with glass stopper and the 
polymerization was thereafter initiated by placing the tubes in a water bath heated to $50{ }^{\circ} \mathrm{C}$. After $24 \mathrm{~h}$ the resulting composite beads were transferred to $50 \mathrm{~mL}$ polypropylene centrifugation tubes followed by addition of the etching solution $\left(3 \mathrm{M} \mathrm{NH}_{4} \mathrm{HF}_{2}\right.$ in water). The tubes were subsequently shaken on a rocking table for $24 \mathrm{~h}$. Thereafter, the polymers were washed with $\mathrm{MeOH} / 1 \mathrm{M} \mathrm{HCl}(80: 20 \mathrm{v} / \mathrm{v})(3 \times 40 \mathrm{~mL})$ and solvent extracted in a Soxhlet apparatus with $\mathrm{MeOH}$ for $24 \mathrm{~h}$. The resulting polymer beads (MIP-M, NIP-M) were dried in vacuo overnight.

Hierarchically imprinted microspheres (MIP-H, NIP-H). Fmoc-pTyr@Si-(A-E) (1.0 g each) were each soaked in dry ACN (2 $\mathrm{mL}$ ) in $50 \mathrm{~mL}$ Schenk tubes. Next, PMP and functional monomer 1 (both 2 eq. with respect to template loading) were added and the solvent thereafter removed under vacuum. Next, the silica samples were deaerated (three cycles vacuum- $\mathrm{N}_{2}$ ) and kept under continuous $\mathrm{N}_{2}$ flow. A monomer mixture consisting of acrylamide (28 mg, $0.39 \mathrm{mmol})$, PETA (1.55 g, $5.20 \mathrm{mmol}$ ) and ABDV (1\% w/w based on total monomers) dissolved in acetonitrile $(2.4 \mathrm{~mL})$ was prepared. The silica samples were thereafter soaked in $750 \mu \mathrm{L}$ of the monomer mixture. For the nonimprinted material the monomer mixture $(750 \mu \mathrm{L})$ was mixed with urea monomer (the same amount as for corresponding MIP) prior to addition to the silica. The tubes were closed with glass stoppers and placed in a water bath heated to $50{ }^{\circ} \mathrm{C}$ for $24 \mathrm{~h}$. Next, the materials were submitted to etching, washing and solvent extraction as in the case of MIP-M and NIP-M described above. Five hierarchically imprinted MIPs (MIP-HAMIP-HE) and corresponding NIPs (NIP-HA-NIP-HE) were obtained (Table S2†).

\section{Solid phase extraction}

Polymer particles $(20 \mathrm{mg})$ were packed in single fritted SPE cartridges (ISOLUTE, Biotage) and protected with a frit on top. The polymers were first conditioned with $95 \% \mathrm{ACN}+0.1 \% \mathrm{FA}(2$ $\times 1 \mathrm{~mL}$ ) followed by loading of an equimolar mixture of FmocpTyr-OH, Fmoc-pSer-OH and Fmoc-Tyr-OH (1 mL, each at 100 $\mu \mathrm{M}$ concentration) in $95 \% \mathrm{ACN}+0.1 \% \mathrm{FA}$. The washing step was performed with $80 \% \mathrm{ACN}+0.1 \% \mathrm{FA}(1 \mathrm{~mL})$ and it was followed by elution with $80 \% \mathrm{MeOH}+1 \%$ TFA $(1 \mathrm{~mL})$. Flowthrough (FT), washing (W) and elution (E) fractions were analyzed by reversed phase HPLC to determine the unbound amount of each compound. The column was Synergi $4 \mu \mathrm{m}$ POLAR-RP $80 \AA$ (Phenomenex, $75 \times 2 \mathrm{~mm}$ ). Mobile phases were (A) $\mathrm{H}_{2} \mathrm{O}+0.1 \%$ TFA and (B) $\mathrm{MeOH}+0.1 \%$ TFA. A linear gradient method of $40 \%$ B to $80 \%$ B in $15 \mathrm{~min}$ at a flow rate of $0.6 \mathrm{~mL}$ $\min ^{-1}$ was used. The injection volume was $20 \mu \mathrm{L}$ and the detection was performed by UV absorbance measurement at $265 \mathrm{~nm}$. The resulting peak areas were used to calculate the amount of bound analytes $(B)$ on the polymer according to eqn (1),

$$
B=\left(C_{0}-c\right) v / m
$$

where $C_{0}$ is the initial solute concentration, $c$ is the final free solute concentration in the supernatant, $v$ is the total volume of the adsorption mixture, and $m$ is the mass of polymer in each vial. The results are averages of three independent experiments.

\section{Binding isotherms}

The polymers (10 $\mathrm{mg}$ each) were separately mixed with $0.5 \mathrm{~mL}$ of Fmoc-pTyr-OH at 0.05, 0.1, 0.25, 0.50, 1.0 and $1.5 \mathrm{mM}$ concentration in $95 \%$ ACN and shaken vigorously for $2 \mathrm{~h}$. Next, the samples were centrifuged and the supernatant analyzed by reversed phase HPLC using method described above to determine concentration of unbound Fmoc-pTyr-OH. The amount of bound amino acid per unit mass of polymer $(B)$ was calculated according to eqn (1). Each experiment was performed three times. Binding curves were constructed by plotting $B$ against free concentration $c$ and were subsequently fitted by non-linear regression in the GraphPad Prism 7 software (GraphPad Software, La Jolla, CA, USA) to a Langmuir mono-site model:

$$
B=B_{\max } c /\left(K_{\mathrm{d}}+c\right)
$$

where $B_{\max }$ is the maximum amount of solute bound by the polymer particles at saturation. The association constants $K_{\mathrm{a}}$ were calculated as the inverse of the dissociation constants $\left(K_{\mathrm{d}}\right)$.

\section{Equilibrium binding tests}

The polymers (10 $\mathrm{mg}$ each) were suspended in $0.5 \mathrm{~mL}$ of a mixture of VPYI, VPSI and VYI (each $20 \mu \mathrm{M}$ ) in $95 \%$ ACN + $0.1 \% \mathrm{FA}$ and shaken vigorously for $2 \mathrm{~h}$. Next the samples were centrifuged and the supernatant $(400 \mu \mathrm{L})$ was dried (Genevac EZ-2 evaporator), dissolved in 15\% ACN $(200 \mu \mathrm{L})$ and analyzed by reversed phase HPLC. The column was Prodigy $5 \mu \mathrm{m}$ ODS-3 $100 \AA$ A (Phenomenex, $150 \times 4.6 \mathrm{~mm}$ ). Mobile phases were (A) $\mathrm{H}_{2} \mathrm{O}+0.1 \%$ TFA and (B) ACN $+0.1 \%$ TFA. A linear gradient method of $15 \%$ B to $20 \%$ B in $10 \mathrm{~min}$ at a flow rate of $1.5 \mathrm{~mL}$ $\min ^{-1}$ was used. The injection volume was $100 \mu \mathrm{L}$ and the detection was performed by UV absorbance measurement at $210 \mathrm{~nm}$. The amount of bound peptide per unit mass of polymer (B) was calculated according to eqn (1). Each experiment was performed three times. The test was repeated for octapeptides, using instead a mixture of DRVPYIHPF, DRVPSIHPF and DRVYIHPF (each $20 \mu \mathrm{M}$ ) in 95\% ACN $+0.1 \%$ TFA. The method for HPLC analysis was a linear gradient of $20 \%$ B to $30 \%$ B in 10 min with other parameters remaining unchanged.

\section{Extraction of phosphopeptides from protein digest}

Protein digestion. The twelve proteins, three thereof phosphorylated on serine residues ( $\alpha$-casein, $\beta$-casein and ovalbumin), were separately dissolved in TEAB $(50 \mathrm{mM})$ to concentrations of $20 \mathrm{pmol} \mu \mathrm{L}^{-1}$. Proteins were reduced with $10 \mathrm{mM}$ DTT at $56^{\circ} \mathrm{C}$ for $30 \mathrm{~min}$. The proteins were subsequently alkylated with $40 \mathrm{mM}$ IAA at room temperature for $30 \mathrm{~min}$ kept in dark. The proteins were digested with trypsin $(1 \%, w / w)$ at $37{ }^{\circ} \mathrm{C}$ for $12 \mathrm{~h}$. The protein digest sample was prepared by mixing the peptides originating from the proteins in equimolar ratio and dilution with $0.1 \%$ TFA to reach final concentration of $1 \mathrm{pmol} \mu \mathrm{L}^{-1}$. The mixture was stored at $-20{ }^{\circ} \mathrm{C}$ until further use.

The protein digest $\left(20 \mu \mathrm{L}, 1 \mathrm{pmol} \mu \mathrm{L}^{-1}\right)$ and peptide mixture ( $5 \mu \mathrm{L}, 1$ pmol $\mu \mathrm{L}^{-1}$ of each peptide, see Table 2 ) were diluted to 1 $\mathrm{mL}$ with loading solvent (95\% ACN $+1 \% \mathrm{FA})$ (diluted digest). Polymers ( $2 \mathrm{mg}$ each) were mixed with $100 \mu \mathrm{L}$ of diluted digest 
and agitated in a Thermomixer at $25{ }^{\circ} \mathrm{C}(1300 \mathrm{rpm})$ for $30 \mathrm{~min}$. The polymer suspension was transferred to a pipette tip microcolumn $(200 \mu \mathrm{L})$ protected with a C8 plug and passed through the column with the aid of a syringe and the flowthrough fraction was collected. Thereafter washing was performed with $95 \% \mathrm{ACN}+1 \% \mathrm{FA}(100 \mu \mathrm{L})$. The flow-through and washing fractions were pooled. Finally elution was performed with $80 \% \mathrm{MeOH}+0.1 \%$ TFA $(100 \mu \mathrm{L})$ and the elution fraction saved. The combined flow-through and washing (FTW) and elution (E) fractions and a fraction of the sample before MIP treatment were dried in a speedvac and redissolved in $0.1 \%$ TFA $(5 \mu \mathrm{L})$. Each sample $(0.5 \mu \mathrm{L})$ was spotted together with the DHB matrix $(0.5 \mu \mathrm{L})$ on a MALDI target plate, dried and analyzed by MALDI-TOF/TOF mass spectrometry.

\section{Results and discussion}

\section{Preparation of materials}

The purpose of the study was to investigate the influence of porosity parameters in phosphotyrosine (pY) imprinted materials on their static and dynamic peptide binding properties. We therefore decided to compare the performance of our previously reported pY-MIP (used as benchmark) featuring a broad pore size distribution, with controlled pore size formats based on silica templating (Fig. 1).

The benchmark polymer was prepared using the functional monomer 1 and the bis-PMP salt of Fmoc-pTyr-OEt dissolved in $\mathrm{ACN}$ in a $2: 1$ stoichiometric ratio. Acrylamide was added as a supplementary monomer to provide additional hydrogen bond stabilization and pentaerythritoltriacrylate (PETA) was used as crosslinking monomer. Conventional azo-initiated thermal polymerization at $50{ }^{\circ} \mathrm{C}$ subsequently afforded the imprinted and non-imprinted polymers which were subsequently crushed and sieved to a $25-36 \mu \mathrm{m}$ particle size fraction. The particles were subjected to template removal by washing
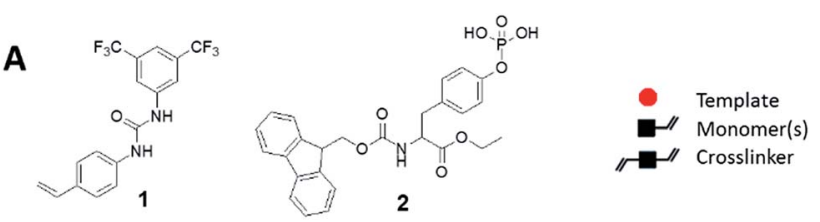

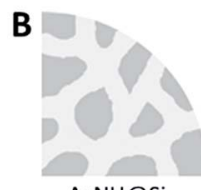
AcNH@Si

C

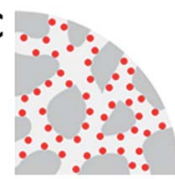

Fmoc-pTyr@Si-A-E

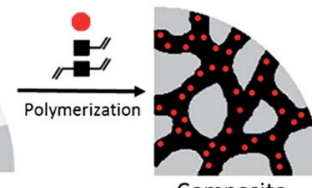

Composite

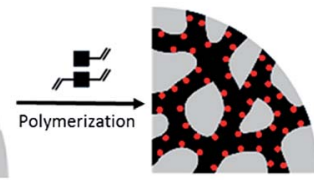

Composite
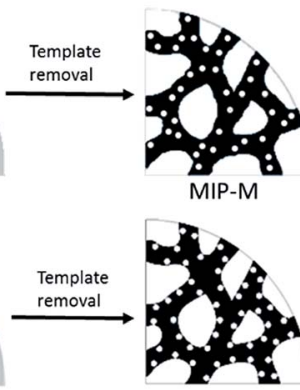

MIP-HA - MIP-HE
Fig. 1 (A) Structures of urea-based functional monomer (1) and Fmoc-pTyr-OEt template (2). Schematic representation of preparation of (B) MIP-M and (C) hierarchically imprinted MIPS (MIP-HA-MIP-HE). with acidified $\mathrm{MeOH}$, followed by extraction with $\mathrm{MeOH}$ and drying.

The silica-templated polymers were subsequently prepared following two alternative approaches (Fig. 1). In both approaches we used mesoporous $\left(D_{\mathrm{p}} \approx 50 \mathrm{~nm}\right)$ spherical silica microparticles as vessels for polymerization in two variants i.e. with free or immobilized template. In the former approach (Fig. 1B) endcapped silica was allowed to soak in the prepolymerization mixture (identical to the one used to prepare the benchmark polymer i.e. containing pY template, monomers and initiator) whereafter the particles were thermally cured at $50{ }^{\circ} \mathrm{C}$. This contrasted with the latter approach (Fig. 1C) where the template had been pre-immobilized on the surface of the silica pore walls (Fig. S1 $\dagger$ ). After $24 \mathrm{~h}$ curing the silica mold was dissolved by treatment with an aqueous solution of $\mathrm{NH}_{4} \mathrm{HF}_{2}$ leaving behind organic polymer beads with a size and morphology reflecting those of the original silica (Fig. S3 and S4 $\dagger$ ).

A crucial parameter in the latter approach is the surface coverage with immobilized template. A high coverage may lead to template clustering which precludes the formation of separate binding sites whereas a low coverage leads to materials featuring insufficient binding capacity. We therefore studied this parameter in more detail and compared polymers prepared from five different silica templates with increasing template coverages (Table $\mathrm{S} 1 \dagger$ ).

The polymers were characterized by elemental analysis (Table S3†), optical and scanning electron microscopy (SEM) (Fig. S3 and S4 $\dagger$ ), IR spectroscopy (Fig. S6 $\dagger$ ), swelling measurements and nitrogen sorption analysis (Table 1, Fig. S5†). This confirmed their identity and near identical chemical compositions. The SEM images confirmed that the silica-templated materials retained the spherical shape and size of the silica scaffold after etching. This indicates that the residual beads originated from polymer formed in the silica pore system.

Otherwise only the nitrogen sorption technique, which provides information concerning the porous properties of the materials, and associated swelling tests gave evidence for differences between the polymers (Table 1, Fig. S5†).

Thus, all polymers except MIP-B exhibited a mesoporous morphology with surface areas larger than $200 \mathrm{~m}^{2} \mathrm{~g}^{-1}$ and

Table 1 Physical properties of Fmoc-pTyr-OEt imprinted and nonimprinted polymers ${ }^{a}$

\begin{tabular}{lllll}
\hline Polymer & $\begin{array}{l}S \\
\left(\mathrm{~m}^{2} \mathrm{~g}^{-1}\right)\end{array}$ & $\begin{array}{l}V_{\mathrm{p}} \\
\left(\mathrm{mL} \mathrm{g}^{-1}\right)\end{array}$ & $\begin{array}{l}D_{\mathrm{p}} \\
(\mathrm{nm})\end{array}$ & $\begin{array}{l}\text { Swelling } \\
\left(\mathrm{mL} \mathrm{mL}^{-1}\right)\end{array}$ \\
\hline MIP-B & 72 & 0.06 & 3.4 & 2.3 \\
NIP-B & 248 & 0.48 & 8.5 & 1.4 \\
MIP-M & 264 & 0.84 & 12.4 & 2.1 \\
NIP-M & 325 & 1.22 & 15.1 & 1.7 \\
MIP-HE & 326 & 0.94 & 10.4 & 1.9 \\
NIP-HE & 201 & 0.89 & 17.0 & 1.9
\end{tabular}

${ }^{a}$ The BET specific surface area $(S)$, specific pore volume $\left(V_{\mathrm{p}}\right)$ and average pore diameter $\left(D_{\mathrm{p}}\right)$ were calculated from the nitrogen adsorption isotherms whereas the swelling was determined by soaking $100 \mathrm{mg}$ of packed bed of polymer particles in ACN as described in the experimental section. 
relatively narrow pore size distributions (Fig. S5†) with average pore diameters exceeding $10 \mathrm{~nm}$. This contrasted with MIP-B, featuring nearly no dry state porosity but significant swelling in acetonitrile. In view of the contrasting MIP/NIP properties in this case, the origin of this effect is template related. It has previously been attributed to a template induced delay in the phase separation. ${ }^{9}$

We gratefully noted that the silica-templated materials featured significantly increased surface areas and average pore sizes. It remained to be investigated how this morphology difference translated into the peptide binding properties of the materials.

\section{Binding isotherms and imprinting efficiency}

In order to compare the affinity and capacity of the materials we recorded the binding isotherms of all materials for the template analogue Fmoc-pTyr-OH in 95\% ACN (Fig. 2A and S2 $\dagger$ ). This solvent was also used in the polymerization step and will hence promote polymer chain conformers present during imprinting, this in turn enhancing imprinting efficiency. Generally, binding to the imprinted polymers exceeded by far that to the corresponding non-imprinted polymers, the latter displaying binding capacities not exceeding $8 \mu \mathrm{mol} \mathrm{g}^{-1}$. Comparing different isotherm models ${ }^{23}$ it was clear from the correlation coefficients that the data for all imprinted polymers fitted well to a $1: 1$ binding model (Table $\mathrm{S} 4 \dagger$ ). Hence, the imprinted sites appear uniform in this concentration interval. ${ }^{24}$ This is in agreement with imprinting stemming from a near stoichiometric monomer-template complex formation. Indeed, ${ }^{1} \mathrm{H}$ NMR titrations has revealed strong interactions between the two species with $K_{\mathrm{a}}$ exceeding $10000 \mathrm{M}^{-1}$.

Fig. 2C shows the binding constants and capacity estimated by non-linear curve fitting of the data in Table S4† assuming
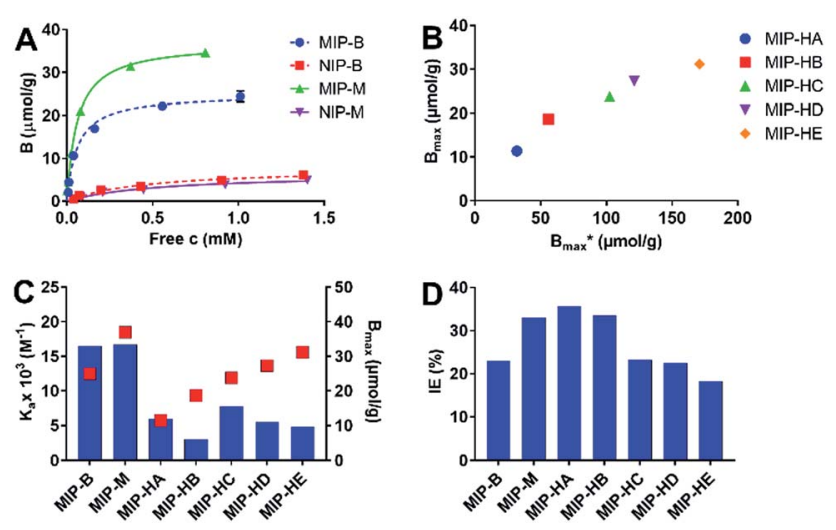

Fig. 2 (A) Binding isotherms of Fmoc-pTyr-OH (concentration range 0.05-1.5 mM) for MIP-B (blue circles), NIP-B (red squares), MIP-M (green triangles), NIP-M (purple triangles). The results are averages of three replicates with error bars representing standard deviation. (B) Nominal $\left(B_{\max }{ }^{*}\right)$ versus measured $\left(B_{\max }\right)$ capacity of MIPs. (C) Association constants $\left(K_{\mathrm{a}}\right.$, blue bars) and binding capacities ( $B_{\max }$, red squares) for Fmoc-pTyr-OH interacting with imprinted polymers in $95 \%$ ACN. The binding parameters were obtained by fitting of the binding data in (A) and Fig. $\mathrm{S} 2 \uparrow$ to a Langmuir mono-site binding model. (D) Imprinting efficiency (IE) of MIPs. a Langmuir mono-site binding model. The silica-templated polymer MIP-M prepared using free template displayed the most promising performance after ranking of the polymers in terms of both binding constant and saturation capacity (Fig. 2C). This is likely to be the result of a more open pore system (Table 1) offering improved accessibility for the guest to the imprinted sites. However, a direct comparison of saturation capacities may be misleading given the different template loads used when synthesizing the materials. Instead it is interesting to compare the imprinting efficiency which relates the experimentally determined capacity to the nominal value based on the actual load of template. The silica-templated materials (e.g. MIP-M, MIP-HA) show the highest values approaching $35 \%$ imprinting efficiency, to be compared with an efficiency of $23 \%$ for the benchmark material MIP-B (Fig. 2D, Table S4 $\dagger$ ). Turning to the hierarchically imprinted materials two striking features should be noted. First of all, the template immobilization seems to compromise the binding affinity as seen in the $c a$. three times lower binding constants for these materials compared to the benchmark MIP-B and MIP-M. Immobilization prevents imprinting memory with respect to the C-terminal substituent of the template. Nevertheless, the absence of a clear trend when comparing MIP-H(AE) indicates that the quality of the binding sites are similar for these materials, also in agreement with the strong monomer template interactions (vide supra). The second striking feature is the apparent correlation between the measured capacity and the nominal value (Fig. 2B). Also this observation is in agreement with the presence of stable monomer template complexes. It is interesting to note that the efficiency decreases with template coverage. This we attribute to template clustering and site coalescence as a result thereof.

\section{Investigation of binding selectivity}

In order to probe the binding selectivity of the materials a competitive solid-phase extraction (SPE) experiment was performed. An equimolar mixture of three amino acids (Fmoc-pTyr$\mathrm{OH}$, Fmoc-pSer-OH and Fmoc-Tyr-OH) was loaded onto each material packed in SPE cartridges (see experimental part) followed by a washing and an elution step. The amount of each analyte in each fraction was quantified by reversed phase HPLC analysis (Fig. 3 and $\mathrm{S} 7 \dagger$ ). Non-imprinted materials showed an overall weak retention of the analytes, all of which were quantitatively recovered in the flow-through and wash fractions (Fig. S7 $\dagger$ ). Nevertheless, as can be seen in the wash fraction recoveries, a weak preference for the phosphorylated analytes, notably pY, was present. This effect was clearest for the materials prepared in presence of free template (MIP-B, MIP-M). When considering the imprinted materials all of them showed a pronounced selectivity for phosphorylated amino acids with a clear preference for phosphotyrosine. MIP-B, MIP-M and MIP$\mathrm{H}(\mathrm{C}-\mathrm{E})$ retained phosphotyrosine very strongly while phosphoserine was mainly found in the wash fractions. The retention of phosphotyrosine on the hierarchically imprinted materials increased with the order of increasing template load, a result corroborated by the measured saturation capacities. 

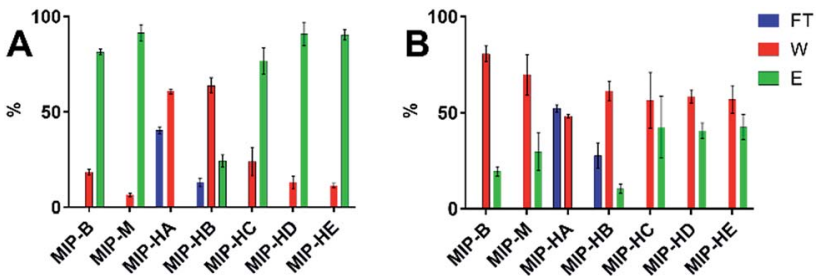

Fig. 3 SPE test results for Fmoc-pTyr-OH (A) and Fmoc-pSer-OH (B) showing \% of each analyte in flow-through (FT), washing (W) and elution (E) fractions. The following conditions were applied: loading $95 \% \mathrm{ACN}+0.1 \% \mathrm{FA}$, washing $80 \% \mathrm{ACN}+0.1 \% \mathrm{FA}$, elution $80 \% \mathrm{MeOH}$ $+1 \%$ TFA. The bars show the average of three replicas and the error bars represent standard deviation.

To gain insight into the potential phosphopeptide preference of the materials we designed a model system based on the peptide hormone angiotensin II (DRVYIHPF) including different phosphorylated analogues and truncated sequences. The ability of the receptors to discriminate $\mathrm{pY}$ and $\mathrm{pS}$ in the same sequence context and in peptides of different sizes was thus investigated by comparing the retention of the phosphorylated peptides DRVpYIHPF, DRVpSIHPF, VPYI, VpSI, with the nonphosphorylated peptides DRVYIHPF and VYI.

We first investigated binding of the shorter sequences VpYI, VPSI and VYI. A ternary mixture of these peptides was thus equilibrated with each of the materials followed by measurement of bound and unbound fractions (Fig. 4). The uptakes measured for the imprinted materials showed a pronounced phosphopeptide preference, in the case of MIP-B and MIP-M accompanied by a notable pY selectivity. An interesting reversal of the binding preference was observed for all the nonimprinted materials which exhibited a strong pS selectivity. We believe this to be related to the different basicities of the alkyl versus aryl phosphate groups (pY: $\mathrm{p} K_{2}=5.80 ; \mathrm{pS}$ : $\mathrm{p} K_{2}=6.19$ ).

Turning to the native octapeptides (DRVPYIHPF, DRVPSIHPF and DRVYIHPF) we narrowed the investigation to include MIP-B, MIP-M and MIP-HE. Equilibrium binding tests using an equimolar mixture of the three peptides led to the binding results shown in Fig. 5 .

Considering first the uptakes measured for the pY peptide we note that these are significantly lower on the imprinted materials prepared using free template (MIP-B and MIP-M: $c a$. 22 and $18 \%$ respectively) compared to the uptakes displayed by

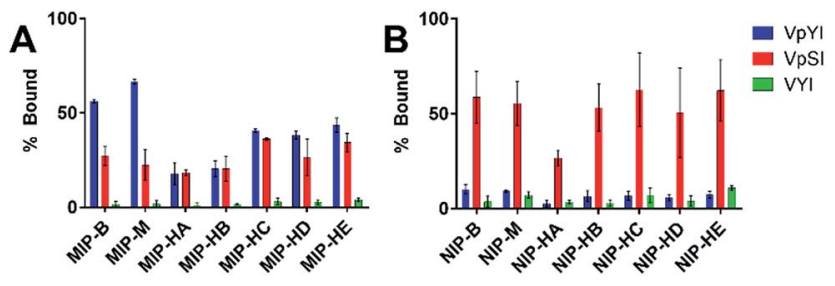

Fig. 4 Results of binding equilibrium test with equimolar mixture of $\mathrm{VpYI}, \mathrm{VpSI}$ and $\mathrm{VYI}$ in $95 \% \mathrm{ACN}+0.1 \% \mathrm{FA}$ for (A) MIPs and (B) NIPs. The bars show the average of three replicas and the error bars represent standard deviation.

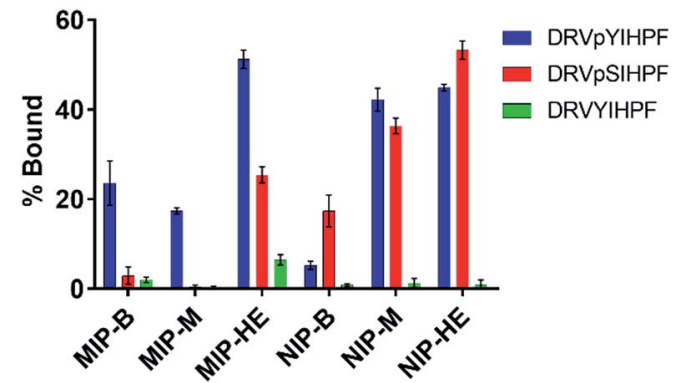

Fig. 5 Results of binding equilibrium test of equimolar ternary mixture of DRVPYIHPF, DRVpSIHPF and DRVYIHPF in 95\% ACN + 0.1\% TFA. The bars show the average of three replicas and the error bars represent standard deviation.

the hierarchically surface imprinted material (MIP-HE: 50\%). Moreover, the uptakes shown by the former materials are lower than those measured for the tripeptides. All in all, this indicates that the binding sites in these materials are more buried and therefore less accessible. This also explains the high binding specificity which is reflected in the lack of uptake of the pS peptide. On the other hand, MIP-HE, featuring the surface imprinted sites, did bind this peptide, albeit to a lower extent than the pY peptide. The non-imprinted materials NIP-M and NIP-HE showed either no side chain selectivity or a preference for $\mathrm{PS}$ peptide (NIP-B).

\section{Phosphopeptide enrichment from complex peptide mixtures}

To validate the ability of the controlled pore size materials to enrich phosphopeptides from complex peptide mixtures we turned to protein digests. Hence, a sample originating from the tryptic digestion of twelve proteins (of which three contain a high abundance of serine-phosphorylated amino acids i.e. ovalbumin, $\alpha$-casein and $\beta$-casein ${ }^{25}$ was spiked with eight $\mathrm{pY}$ peptides one pS peptide and two Y peptides (Table 2). The size of the peptides ranged from 8 to 31 amino acids with charges spanning from -5 to +0.1 and GRAVY index from -1.27 to -0.26 (for the parent nonphosphorylated peptides). Phosphotyrosine peptides represented less than $1 \%$ (by mass) of all peptides in the sample. The polymers $(2 \mathrm{mg})$ were equilibrated with the digest sample in the loading buffer for 30 minutes. Thereafter, the particle suspensions were transferred with pipette to tip columns and the packing then protected with a C8 plug. The flow-through (FT) fraction was collected and pooled with the fraction collected in the washing step (W). Thereafter, elution was performed and the resulting fraction (E) collected and saved. All fractions were dried, redissolved and analyzed by MALDI-MS with respect to the presence of tyrosine-, serine- and non-phosphorylated peptides.

As can be seen in Fig. 6A, five out of the eight spiked pY peptides along with four $\mathrm{pS}$ peptides from the protein digest and the spiked $\mathrm{pS}$ peptide were identified in the crude sample before enrichment (Fig. 6A, Table S5 $†$ ). The sample clean-up performed with the MIPs allowed the reduction of ion signals stemming from nonphosphorylated peptides to a great extent (Fig. 6B-D, Table S5 $\dagger$ ) for improved detection of phosphopeptides. 
Table 2 Model peptides spiked in the tryptic digest of twelve proteins

\begin{tabular}{lllll}
\hline Peptide sequence & Size $^{a}$ & {$[\mathrm{M}+\mathrm{H}]^{+}$} & Net charge $^{b}$ & GRAVY index $^{c}$ \\
\hline DRVYIHPF & 8 & 1046.54 & 0.1 & -0.32 \\
DRVPSIHPF & 8 & 1050.48 & 0.1 & -1 \\
GADDSYYTAR & 10 & 1118.47 & 0.1 & -1.24 \\
DRVPYIHPF & 8 & 1126.51 & -1 & -0.32 \\
GADDSYPYTAR & 10 & 1198.44 & -1 & -1.24 \\
GADDSpYPYTAR & 10 & 1278.41 & -1 & -0.98 \\
GSTAENAEPYLR & 11 & 1290.54 & -1 & -1.00 \\
MHLPSPTDSNFPYR & 13 & 1644.68 & -1.27 \\
GSHQISLDNPDPYQQDFFPK & 19 & 2316.00 & -1.25 \\
RPAGSVQNPVYYHNQPLNPAPSRD & 23 & 2594.23 & -2 & -0.55
\end{tabular}

${ }^{a}$ Number of amino acids. ${ }^{b}$ Net charge at pH 7. ${ }^{c}$ GRAVY index for the parent nonphosphorylated peptide.

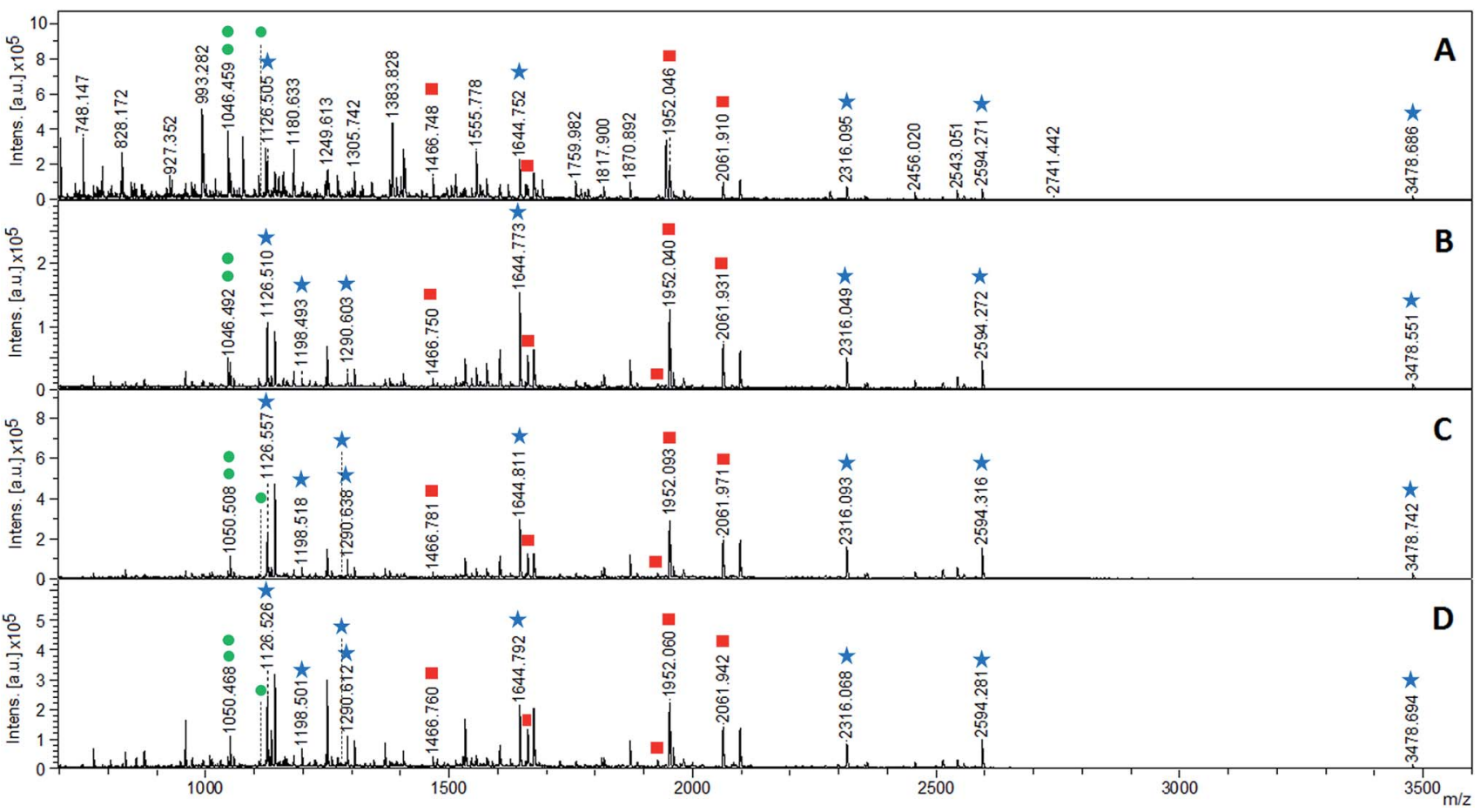

Fig. 6 MALDI mass spectra obtained for peptide mixture before enrichment (A), elution fraction from MIP-B (B), MIP-M (C) and MIP-HE (D). Marked are spiked phosphotyrosine peptides (asterisk), phosphoserine peptides (square) and spiked phosphoserine and nonphosphorylated peptides (circle).

MIP-B was able to extract seven out of the eight spiked pY peptides whereas spherical MIPs (MIP-M and MIP-HE) extracted all spiked pY peptides. The combined flow-through and washing fractions from the MIPs, on the other hand, contained four (MIP-B and MIP-M) or two (MIP-HE) pS peptides and only two (MIP-B, MIP-M) or three (MIP-HE) of the eight pY peptides (Table S5, Fig. S8†).

Peptide ion signal intensities in MALDI-MS are dependent on the ionization efficiency of each of the analytes and the solvent/matrix composition. ${ }^{26}$ This precludes its use for direct quantitative analysis unless special precautions are taken and carefully optimized internal standards are used. Changes in the relative abundance of a given compound can however be determined by comparing normalized signal intensities. ${ }^{26}$ Hence, we calculated the relative signal intensities of the pY, pS and $\mathrm{Y}$ peptides respectively to the total intensity of all selected peptides in a given fraction (Table S6, $\uparrow$ Fig. 7).

The increase in the relative signal intensities of the pY peptides together with a decrease of the nonphosphorylated peptide signals show that the MIPs in principle work as expected and confirm our previous observations. ${ }^{10} \mathrm{~A}$ more refined analysis of the data allows further conclusions concerning the relative performance of the materials. Hence, we compared the normalized signal intensities collectively for all assigned $\mathrm{PY}$ and pS peptides (Fig. 8A) and of all assigned pY and Y peptides (Fig. 8B). This showed that the overall $\mathrm{pY} / \mathrm{pS}$ ratio increased 


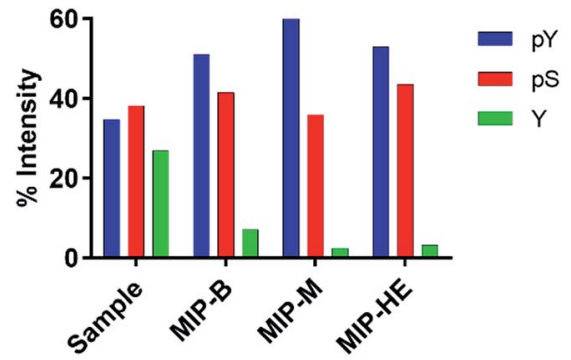

Fig. 7 Normalized signal intensities for the $p Y, p S$ and $Y$ peptides found in the elution fractions given as percentage of accumulated intensity of selected ions in a given fraction.

significantly after enrichment. On the other hand, the highest phosphopeptide specific enrichment factor (pY/Y) was obtained using the templated materials MIP-M and MIP-HE. Hence, the nonphosphorylated peptides were depleted from the sample treated with these MIPs. MIP-M here proved to be the most successful in removing the $\mathrm{Y}$ peptide (angiotensin).

Given the aim of enriching larger phosphopeptide fragments we were interested in how the selectivity and enrichment responded to the size of the peptide. For this purpose we compared groups of peptides (one pY, one pS and one nonphosphorylated peptide) of similar size with the pY being of size $8,14,19$ and 24 amino acids. The octapeptides corresponding to the angiotensin II model system (vide supra) offers the most stringent test for the $\mathrm{pY} / \mathrm{pS}$ selectivity since it features peptides of identical sequences at the $\mathrm{C}$ and $\mathrm{N}$ terminus. In spite of the sequence similarity the MIPs showed a marked pY selectivity $(\mathrm{pY} / \mathrm{pS} \approx 1.5)$ also in the more complex digest sample. Turning to the size dependent phosphospecific enrichment (Fig. 8C and D) we observed clear differences between the materials.

Whereas the benchmark material MIP-B showed the lowest $\mathrm{pY} / \mathrm{Y}$ ratio and only a minor increase of the ratio with peptide size, both the mesoporous materials MIP-M and MIP-HE showed a clear trend of increasing enrichment efficiency with increasing
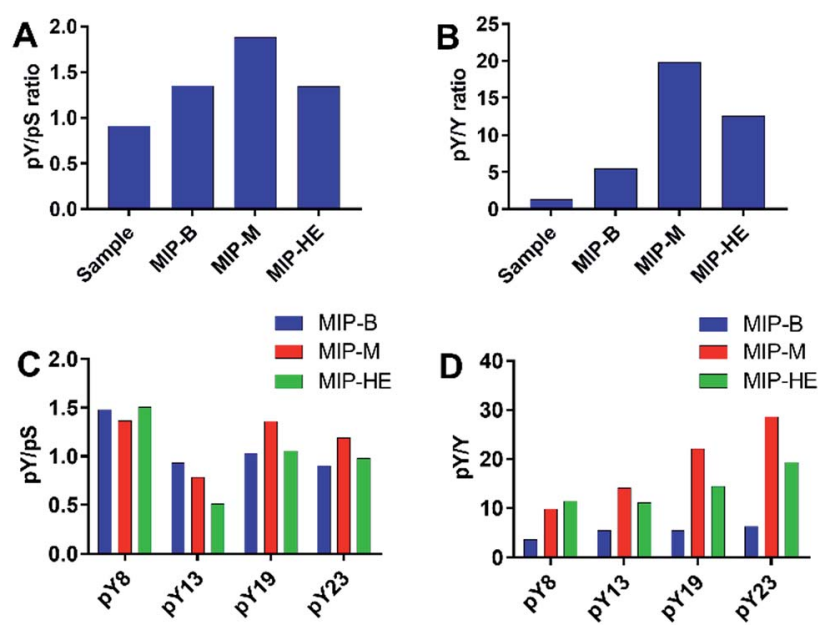

Fig. 8 Ratios of normalized signal intensities of the $p Y, p S$ and $Y$ peptides for nontreated and MIP-treated samples (A and B) and with respect to peptide size for the treated samples ( $C$ and $D)$. size of the peptide. This we attribute to the larger average pore diameter of these materials and a relative increase in the number of sites associated with such pores. In addition to size exclusion effects, it is important to consider the surface chemistry of the materials. The silica template defines not only the average size and distribution of pores in the polymer replica but also the surface chemistry of these pores. Hence, as we have previously shown by the Engelhardt test ${ }^{18}$ surface hydrophilicity is affected by the type of silica surface modification, e.g. use of endcapped silica as template leads to a more hydrophobic material than a material synthesized from a rehydroxylated silica template. The acetamide endcapped silicas used in the current study may have resulted in a more hydrophilic surface compared to that present in the benchmark material. Such material will display a lower degree of nonspecific peptide binding.

\section{Conclusions}

In conclusion, we have shown that silica-templated MIP synthesis and surface imprinting are useful approaches to bias the phosphopeptide preferences to larger peptide sizes $(>2 \mathrm{kDa})$ as well as to suppress nonspecific binding of nonphosphorylated peptides. Further optimization of the synthesis parameters is in progress to adapt the materials to middle down and top down phosphoproteomics workflows.

\section{Acknowledgements}

This work was supported by the EU-funded Marie Curie ITN project PEPMIP (PITN-GA-2010-264699). Work in the ONJ laboratory is supported by a generous grant from the VILLUM Foundation to the VILLUM Center for Bioanalytical Sciences at SDU, Odense.

\section{Notes and references}

1 S. A. Johnson and T. Hunter, Nat. Methods, 2005, 2, 17-24.

2 T. E. Thingholm, T. J. Jorgensen, O. N. Jensen and M. R. Larsen, Nat. Protoc., 2006, 1929-1935.

3 N. M. Riley and J. J. Coon, Anal. Chem., 2016, 88, 74-94.

4 S. J. Humphrey, S. B. Azimifar and M. Mann, Nat. Biotechnol., 2015, 33, 990-995.

5 K. Engholm-Keller and M. R. Larsen, Proteomics, 2013, 13, 910-931.

6 O. Pagel, S. Loroch, A. Sickmann and R. P. Zahedi, Expert Rev. Proteomics, 2015, 12, 235-253.

7 C. J. Tape, J. D. Worboys, J. Sinclair, R. Gourlay, J. Vogt, K. M. McMahon, M. Trost, D. A. Lauffenburger, D. J. Lamont and C. Jørgensen, Anal. Chem., 2014, 86, 10296-10302.

8 B. A. Garcia, J. Am. Soc. Mass Spectrom., 2010, 21, 193-202.

9 M. Emgenbroich, C. Borrelli, S. Shinde, I. Lazraq, F. Vilela, A. J. Hall, J. Oxelbark, E. De Lorenzi, J. Courtois, A. Simanova, J. Verhage, K. Irgum, K. Karim and B. Sellergren, Chem.-Eur. J., 2008, 14, 9516-9529.

10 J. Chen, S. Shinde, M.-H. Koch, M. Eisenacher, S. Galozzi, T. Lerari, K. Barkovits, P. Subedi, R. Krüger, K. Kuhlmann, 
B. Sellergren, S. Helling and K. Marcus, Sci. Rep., 2015, 5, 11438.

11 Y. Chen, D. Li, Z. Bie, X. He and Z. Liu, Anal. Chem., 2016, 88, 1447-1454.

12 L. Xu, Y. Hu, F. Shen, Q. Li and X. Ren, J. Chromatogr. A, 2013, 1293, 85-91.

13 J. Haginaka, H. Tabo and H. Matsunaga, Anal. Chim. Acta, 2012, 748, 1-8.

14 Q. Li, F. Shen, X. Zhang, Y. Hu, Q. Zhang, L. Xu and X. Ren, Anal. Chim. Acta, 2013, 795, 82-87.

15 B. Sellergren and A. J. Hall, in Molecularly imprinted polymers. Man-made mimics of antibodies and their applications in analytical chemistry, ed. B. Sellergren, Elesevier Science B.V., Amsterdam, 2001, vol. 23, pp. 21-57.

16 C. Sulitzky, B. Rückert, A. J. Hall, F. Lanza, K. Unger and B. Sellergren, Macromolecules, 2002, 35, 79-91.

17 M. R. Halhalli, C. S. A. Aureliano, E. Schillinger, C. Sulitzky, M. M. Titirici and B. Sellergren, Polym. Chem., 2012, 3, 10331042.

18 M. R. Halhalli, E. Schillinger, C. S. A. Aureliano and B. Sellergren, Chem. Mater., 2012, 24, 2909-2919.
19 M. M. Titirici, A. J. Hall and B. Sellergren, Chem. Mater., 2003, 15, 822-824.

20 E. Yilmaz, O. Ramström, P. Möller, D. Sanchez and K. Mosbach, J. Mater. Chem., 2002, 12, 1577-1581.

21 J. Hall Andrew, P. Manesiotis, M. Emgenbroich, M. Quaglia, E. De Lorenzi and B. Sellergren, J. Org. Chem., 2005, 70, 1732-1736.

22 S. Kjellström and O. N. Jensen, Anal. Chem., 2004, 76, 51095117.

23 K. A. Connors, Binding constants. The measurement of molecular complex stability, John Wiley \& Sons, New York, 1987.

24 K. D. Shimizu, in Molecularly Imprinted Materials: Science and Technology, 2005, pp. 419-434.

25 M. R. Larsen, T. E. Thingholm, O. N. Jensen, P. Roepstorff and T. J. D. Jørgensen, Mol. Cell. Proteomics, 2005, 4, 873-886.

26 O. Haglund, Qualitative Comparison of Normalization Approaches in MALDI-MS, MSc thesis, Royal Institute of Technology, Stockholm, Sweden, 2008. 